\title{
ESSENTIAL DIMENSION OF INVOLUTIONS AND SUBALGEBRAS
}

\author{
ROLAND LÖTSCHER
}

\begin{abstract}
We use theorems of N. Karpenko about the incompressibility of Severi Brauer varieties and quadratic Weil transfers of Severi Brauer varieties to compute the minimal number of parameters needed to define conjugacy classes of involutions of given type on division algebras of 2-primary index. Similarly we compute the minimal number of parameters needed to define conjugacy classes of étale subalgebras of given type and central simple subalgebras of given degree in division algebras of $p$-primary index.
\end{abstract}

\section{Contents}

1. Introduction 1

2. A general strategy 3

3. Involutions 5

4. Subalgebras $\quad 7$

4.1. Étale subalgebras 8

4.2. Central simple subalgebras 9

5. Essential dimension of related group schemes $\quad 10$

Acknowledgments $\quad 12$

$\begin{array}{ll}\text { References } & 12\end{array}$

\section{INTRODUCTION}

Essential dimension is a certain numerical invariant of algebraic objects measuring their complexity. Roughly, it is defined as the minimal number of independent parameters needed to define algebraic objects of a given type, e.g. degree $n$ central simple algebras, $n$-dimensional quadratic forms, torsors of an algebraic group etc.

Essential dimension was introduced by J. Buhler and Z. Reichstein around 1995 when studying Tschirnhaus transformations of polynomials in one variable. They defined the essential dimension of a finite group $G$ (relative to a base field $F$ ) in invariant theoretic terms and showed that the essential dimension of the symmetric group $G=S_{n}$ can be understood as the minimal number of algebraically independent parameters in a polynomial $p(x)=x^{n}+a_{1} x^{n-1}+\ldots+a_{n}$ obtained by means of Tschirnhaus transformations from the generic degree $n$ polynomial (where

Date: October 20, 2010.

2000 Mathematics Subject Classification. 16W10, 16K20.

Key words and phrases. essential dimension, involutions, central simple algebras, canonical dimension, Severi-Brauer variety, étale algebras. 
$a_{1}, \ldots, a_{n}$ are algebraically independent over $F$ ). Later Reichstein extended this notion to algebraic groups $G$ and showed, that this concept can be used to measure the complexity of algebraic objects like quadratic forms (when $G=\mathbf{O}_{n}$ ), central simple algebras (when $G=\mathbf{P G} \mathbf{L}_{n}$ ), octonion algebras (when $G=\mathbf{G}_{2}$ ) etc.

The most general definition of essential dimension, due to A. Merkurjev, assigns to each (covariant) functor $\mathcal{F}:$ Fields $/ F \rightarrow$ Sets a non-negative integer, called essential dimension of $\mathcal{F}$. We refer to [BF03] and [Me09] for its definition. Briefly, the essential dimension ed $\mathcal{F}$ of the functor $\mathcal{F}$ is less or equal to $n \in \mathbb{N}$ if and only if every element $a \in \mathcal{F}(L)$ (where $L \in$ Fields $/ F$ ) can be defined over a subfield $L_{0} \in$ Fields $/ F$ of transcendence degree at most $n$.

To each functor $\mathcal{F}:$ Fields $/ F \rightarrow$ Sets we can associate its detection functor $\mathcal{D}_{\mathcal{F}}:$ Fields $/ F \rightarrow$ Sets, defined by

$$
\mathcal{D}_{\mathcal{F}}(L)=\left\{\begin{array}{lc}
\emptyset & \text { if } \mathcal{F}(L)=\emptyset \\
\{L\} & \text { otherwise. }
\end{array} .\right.
$$

The essential dimension of $\mathcal{D}_{\mathcal{F}}$ is called canonical dimension of $\mathcal{F}$, denoted cdim $\mathcal{F}$. The concept of canonical dimension was introduced by G. Berhuy and Z. Reichstein [BR05]. See also [Me09]. It is easy to see that $\operatorname{cdim} \mathcal{F} \leq \operatorname{ed} \mathcal{F}$. The fields $L \in$ Fields $/ F$ with $\mathcal{F}(L) \neq \emptyset$ are called splitting fields of $\mathcal{F}$. The functor $\mathcal{F}$ has canonical dimension $\leq n$ if and only if every splitting field $L$ of $\mathcal{F}$ contains a subfield $L_{0} \in$ Fields $/ F$ of transcendence degree $\leq n$ that splits $\mathcal{F}$ as well.

There is a variant of essential (and canonical) dimension relative to a prime number $p$, called essential $p$-dimension (resp. canonical $p$-dimension). Essential $p$ dimension measures the complexity of algebraic objects up to prime to $p$ extensions. We refer to $[\mathrm{Me} 09]$ for its definition.

If $G$ is a group scheme (always assumed of finite type) over $F$ the essential dimension (resp. $p$-dimension) of $G$ is defined as

$$
\text { ed } G=\operatorname{ed} H^{1}(-, G), \quad \text { resp. } \operatorname{ed}_{p} G=\operatorname{ed}_{p} H^{1}(-, G),
$$

where $H^{1}(-, G)$ is the flat cohomology functor, which takes a field extension $L / F$ to the set of isomorphism classes of $G$-torsors over $L$ (in the finitely presented faithfully flat topology).

A variety $X$ over $F$ can be viewed as functor of points

$$
X: \text { Fields } / F \rightarrow \text { Sets, } \quad L \mapsto X(L)=\operatorname{Mor}(\operatorname{Spec} L, X) .
$$

The essential dimension and $p$-dimension of $X$ are easy to compute: They are equal to the dimension of $X$, see [BF03, Proposition 1.17], [Me09, Proposition 1.2]. On the other hand, the canonical dimension (or $p$-dimension) of $X$ is an interesting invariant of $X$ in case that $X$ does not have $F$-rational points. Interesting examples include quadrics, Severi-Brauer varieties and torsors of an algebraic group $G$.

In this paper we are going to make use of the following results due to $\mathrm{N}$. Karpenko:

Theorem 1.1 ([Ka00, Ka09]). Let $D$ be a central division $K$-algebra of degree $p^{r}$ for some $r \geq 0$ and a prime $p$. Then

$$
\operatorname{cdim} \mathrm{SB}(D)=\operatorname{cdim}_{p} \mathrm{SB}(D)=\operatorname{dim} D=p^{r}-1 .
$$


Moreover if $K / F$ is a quadratic separable field extension, $p=2$, and the norm algebra $N_{K / F}(D)$ is split, then

$\operatorname{cdim} R_{K / F}(\mathrm{SB}(D))=\operatorname{cdim}_{2} R_{K / F}(\mathrm{SB}(D))=\operatorname{dim} R_{K / F}(\mathrm{SB}(D))=2 \cdot\left(2^{r}-1\right)$.

We will adopt and freely use the notations and conventions of [KMRT98] with just a few exceptions, that will be made explicit. Most significantly $H^{1}(-, G)$ (and, when $G$ is abelian, $H^{2}(-, G)$ ) will denote flat cohomology rather than Galois cohomology. We refer to $[\mathrm{Wa} 79, \S 17,18]$ for basics on flat cohomology. The difference only shows up for non-smooth group schemes $G$. For example the flat cohomology set $H^{1}\left(F, \mathbf{G O}\left(M_{n}(F), \tau\right)\right.$ ) (where $\tau$ is transposition of matrices) stands in bijection with the set of (all) conjugacy classes of orthogonal involutions $\sigma$ on $M_{n}(F)$, whereas the Galois cohomology set $H_{\text {Gal }}^{1}\left(F, \mathbf{G O}\left(M_{n}(F), \tau\right)\right)$ consists only of those classes, for which $\sigma$ and $\tau$ become conjugate over $F_{\text {sep }}$. If $F_{\text {sep }}$ is not quadratically closed, this is a non-trivial restriction.

The rest of this paper is organized as follows: In section 2 we describe our strategy of applying Karpenko's incompressibility results to the computation of the exact values of the essential dimension (and $p$-dimension) of certain group schemes. In section 3 we apply this strategy to the essential dimension of conjugacy classes of involutions on central simple algebras. In section 4 we consider the essential dimension of conjugacy classes of certain subalgebras of a central simple algebra. We do this in two particular cases. In the first case we consider étale subalgebras of a given type and in the second case we consider central simple subalgebras of given degree. Our main results are given in Theorems 3.1, 4.1 and 4.2.

In section 5 we give close (in certain cases exact) estimates of the essential dimension of group schemes which are related to the previously considered ones.

\section{A general Strategy}

Let $E / F$ be an étale $F$-algebra and $A$ be an Azumaya $E$-algebra. We have an exact sequence

$$
1 \rightarrow R_{E / F}\left(\mathbf{G}_{m}\right) \rightarrow R_{E / F}\left(\mathbf{G L}_{1}(A)\right) \stackrel{\text { Int }}{\rightarrow} \mathbf{A u t}_{E}(A) \rightarrow 1
$$

of group schemes over $F$, where Int takes an element $a$ of $\left(A \otimes_{F} L\right)^{\times}$(for a field extension $L / F)$ to the $E \otimes_{F} L$-algebra automorphism of $A_{L}:=A \otimes_{F} L$ sending $x$ to $a x a^{-1}$. Let $H$ be an arbitrary (closed) subgroup of $\operatorname{Aut}_{E}(A)$ and set $G=\operatorname{Int}^{-1}(H)$. Then we have a commutative diagram

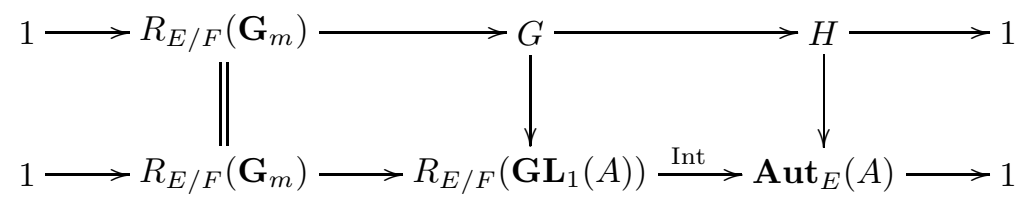

of group schemes over $F$ with exact rows, where the vertical arrows are closed embeddings.

Let $L / F$ be a field extension. The set $H^{1}\left(L, \mathbf{A u t}_{E}(A)\right)$ stands in natural bijection with the set of isomorphism classes of Azumaya $E \otimes_{F} L$-algebras $B$ which become isomorphic to $A_{L}$ over a seperable closure $L_{\text {sep }}$. Its distinguished element is the 
class of $A_{L}$. The group $H^{2}\left(L, R_{E / F}\left(\mathbf{G}_{m}\right)\right)$ is naturally isomorphic to the Brauer group of $E \otimes_{F} L$. The connection map

$$
\delta^{1}: H^{1}\left(L, \operatorname{Aut}_{E}(A)\right) \rightarrow H^{2}\left(L, R_{E / F}\left(\mathbf{G}_{m}\right)\right)=\operatorname{Br}\left(E \otimes_{F} L\right)
$$

takes the isomorphism class of an Azumaya algebra $B$ to the Brauer class of $B \otimes_{E \otimes_{F} L} A_{L}^{\text {op }}=B \otimes_{E} A^{\text {op }}$. Take an $H$-torsor $T$ over some field extension $L$ of $F$. Let $B$ be an Azumaya $L \otimes_{F} E$-algebra representing the image of $T$ in $H^{1}\left(L, \mathbf{A u t}_{E}(A)\right)$. Let

$$
X=R_{E \otimes_{F} L / L}\left(\mathrm{SB}\left(B \otimes_{E} A^{\mathrm{op}}\right)\right) .
$$

The following result will be our main tool for computing essential and canonical dimensions of algebraic groups.

\section{Proposition 2.1.}

$$
\operatorname{cdim} X-\operatorname{dim} H \leq \operatorname{ed} G \leq \operatorname{dim}_{F} A-\operatorname{dim} G
$$

and

$$
\operatorname{cdim}_{p} X-\operatorname{dim} H \leq \operatorname{ed}_{p} G \leq \operatorname{ed} G
$$

for every prime $p$. In particular if $X$ is incompressible (resp. p-incompressible) then ed $G=\operatorname{dim}_{F} A-\operatorname{dim} G\left(\right.$ resp. $\left.\operatorname{ed}_{p} G=\operatorname{ed} G=\operatorname{dim}_{F} A-\operatorname{dim} G\right)$.

Proof. We will first show the inequality $\operatorname{cdim} X-\operatorname{dim} H \leq \operatorname{ed} G$, by adapting [Me09, Theorem 4.8] (cf. [BRV08, Corollary 4.2]) to our situation.

The splitting fields of $X$ are precisely the field extensions $M$ of $L$ for which $T_{M}$ lifts to a $G$-torsor. Let $M$ be a splitting field and let $E$ be a $G$-torsor lifting $T_{M}$, i.e., such that $E *_{G} H \simeq T_{M}$. Let $E_{0}$ be a $G$-torsor over an intermediate field $L \subseteq M_{0} \subseteq M$ such that $\operatorname{tdeg}_{L} M_{0} \leq \operatorname{ed} G_{L} \leq$ ed $G$ and $E_{0} \times_{M_{0}} M \simeq E$. The induced $H$-torsor $T_{0}=E_{0} *_{G} H$ and the $H$-torsor $T_{M_{0}}$ do not need to be isomorphic. However they become isomorphic over an intermediate field $M_{0} \subseteq M_{1} \subseteq M$ of transcendence degree $\operatorname{tdeg}_{M_{0}} M_{1} \leq \operatorname{dim} H$. The field $M_{1}$ is a splitting field of $X$. Since $\operatorname{tdeg}{ }_{L} M_{1} \leq \operatorname{ed} G+\operatorname{dim} H$ this proves the inequality $\operatorname{cdim} X-\operatorname{dim} H \leq \operatorname{ed} G$. The inequality $\operatorname{cdim}_{p} X-\operatorname{dim} H \leq \operatorname{ed}_{p} G$ is obtained with standard modifications of the above argument.

The inequality ed $G \leq \operatorname{dim}_{F} A-\operatorname{dim} G$ follows from [Me09, Corollary 4.3] and the fact that $G$ embeds in the special group scheme $R_{E / F}\left(\mathbf{G L}_{1}(A)\right)$ of dimension $\operatorname{dim}_{F} A$. The inequalites $\operatorname{ed}_{p} G \leq \operatorname{ed} G$ and $\operatorname{cdim}_{p} H \leq \operatorname{cdim} H \leq \operatorname{dim} H$ are clear. For the last claim note that $\operatorname{dim} X-\operatorname{dim} H=\operatorname{dim}_{F} \bar{A}-\operatorname{dim} G$.

Interesting subgroups of $\mathbf{A u t}_{E}(A)$ are actually easy to find: Just endow $A$ with some additional structure (an involution, a quadratic pair, an étale or central simple subalgebra in our examples) and take the subgroup of $\mathbf{A u t}_{E}(A)$ consisting of $E$ algebra automorphisms preserving that additional structure.

Our goal now is to find interesting examples where $X=R_{E \otimes_{F} L / L}\left(\mathrm{SB}\left(B \otimes_{E} A^{\mathrm{op}}\right)\right)$ is in fact $p$-incompressible. We will do this in case that $E / F$ is either a separable quadratic field extension (and $p=2$ ) or $E=F$ (and $p$ is arbitrary). We assume that $\operatorname{deg} A$ is a power of $p$. Note that in these cases in view of Theorem 1.1 all we need for $p$-incompressibility of $X$ is that $D=B \otimes_{E} A^{\text {op }}$ is a division algebra and that $N_{E \otimes_{F} L / L}(D)$ splits when $[E: F]=2$.

We fix a prime $p$ and use the following notation (which slightly differs from the notation used in [KMRT98]): 
Definition 2.1. Let $L$ be a field.

Case char $L \neq p$ : Let $\zeta$ be a primitive $p$ th root of unity in $L$ (we assume its existence) and let $a, b \in L^{\times}$. We denote by $(a, b)_{L}$ the cyclic $L$-algebra generated by symbols $u$ and $v$ with relations $u^{p}=a, v^{p}=b$ and $v u=\zeta u v$.

Case char $L=p$ : Let $a, b \in L$ with $b$ invertible. We denote by $(a, b)_{L}$ the cyclic $L$-algebra generated by symbols $u$ and $v$ with relations $u^{p}-u=a, v^{p}=b$ and $v u=u v+u$.

Note that the definition of $(a, b)_{L}$ in the first case depends on the choice of $\zeta$. For us this choice will not matter, hence we do not include it in the notation. Different choices of $\zeta$ for different algebras $(a, b)_{L}$ will be fine for us, too. The following lemma will help us to produce examples where $D=B \otimes_{E} A^{\text {op }}$ is a division algebra:

Lemma 2.1. Let $a_{1}, b_{1}, \ldots, a_{r}, b_{r}$ be algebraically independent variables over $F$ and let $L=F^{\prime}\left(a_{1}, b_{1}, \ldots, a_{r}, b_{r}\right)$, where $F^{\prime}$ is $F$ adjoined a primitive pth root of unity if $\operatorname{char} F \neq p$ resp. $F^{\prime}=F$ if $\operatorname{char} F=p$. Let

$$
B_{0}=\left(a_{1}, b_{1}\right)_{L} \otimes_{L}\left(a_{2}, b_{2}\right)_{L} \otimes_{L} \cdots \otimes_{L}\left(a_{r}, b_{r}\right)_{L} .
$$

Let $E / F$ be a (finite) separable field extension which is linearly disjoint from $F^{\prime}$ and let $A$ be a central simple E-algebra of p-power degree. Set $M=E \otimes_{F} L=$ $\left(E \otimes_{F} F^{\prime}\right)\left(a_{1}, b_{1}, \ldots, a_{r}, b_{r}\right)$. Then $B_{0} \otimes_{F} A^{\text {op }}$ is a central simple $M$-algebra with

$$
\text { ind } B_{0} \otimes_{F} A^{\text {op }}=p^{r} \text { ind } A \text {. }
$$

Proof. First of all note that ind $A \otimes_{F} F^{\prime}=$ ind $A$ since $F^{\prime} / F$ has degree prime to $p$. Hence we may replace $F^{\prime}$ by $F, A$ by $A \otimes_{F} F^{\prime}$ and $E$ by the field $E \otimes_{F} F^{\prime}$ and hence assume $F^{\prime}=F$. Using induction on $r \geq 0$ one easily reduces to the case $r=1$. The claim then follows from the index formula given in [Me10, (11) §4.1] (cf. [JW90, Prop. 1.15(a)] and [Ti78, Prop. 2.4]).

We summarize this section in a corollary, which will later be invoked for various choices of group schemes $H$.

\section{Corollary 2.1.}

Let either $E=F$ or $E / F$ be a separable quadratic field extension and let $A$ be a division E-algebra of degree $p^{r}$. If $[E: F]=2$ assume that $p=2$ and that $N_{E / F}(A)$ splits. Let $L / F$ and $B_{0}$ be as in Lemma 2.1 and set $B=B_{0} \otimes_{F} E$.

Assume that the class of $B$ lies in the image of $H^{1}(L, H) \rightarrow H^{1}\left(L, \mathbf{A u t}_{E}(A)\right)$ for a subgroup $H$ of $\mathbf{A u t}_{E}(A)$. Then

$$
\text { ed } G=\operatorname{ed}_{p} G=\operatorname{dim}_{F} A-\operatorname{dim} G
$$

where $G$ is the inverse image of $H$ under $\operatorname{Int}: R_{E / F}\left(\mathbf{G L}_{1}(A)\right) \rightarrow \operatorname{Aut}_{E}(A)$.

Proof. Lemma 2.1 implies that $B \otimes_{E} A^{\text {op }}=B_{0} \otimes_{F} A^{\text {op }}$ is a division algebra. In case $[E: F]=2$ note that $N_{E \otimes_{F} L / L}\left(B \otimes_{E} A^{\text {op }}\right)$ splits since $N_{E / F}(A)$ and $N_{E \otimes_{F} L / L}(B)$ split. Theorem 1.1 shows that $X=R_{E \otimes_{F} L / L}\left(\mathrm{SB}\left(B \otimes_{E} A^{\mathrm{op}}\right)\right)$ is $p$-incompressible. Hence the claim follows from Proposition 2.1 .

\section{INVOLUTIONS}

Let $A$ be a central simple $E$-algebra admitting an involution $\sigma$. Let $F=E^{\sigma} \subseteq E$ be the fixed field of central elements under $\sigma$. We call $(A, \sigma)$ a central simple $F$ algebra with involution. For unitary involutions we include the case $E=F \times F$ in 
this definition by allowing $A$ to be a direct product $A=A_{1} \times A_{2}$ with $A_{1}$ and $A_{2}$ central simple $F$-algebras of the same degree.

Two involutions $\sigma^{\prime}$ and $\sigma$ on $A$ are said to be conjugate if there exists $a \in A$ such that $\sigma^{\prime}=\operatorname{Int}(a) \circ \sigma \circ \operatorname{Int}(a)^{-1}$. Equivalently $(A, \sigma)$ and $\left(A, \sigma^{\prime}\right)$ are isomorphic as $F$-algebras with involution.

Conjugate involutions are necessarily of the same type (unitary, symplectic or orthogonal). When $F$ is algebraically closed there are precisely 3 conjugacy classes of involutions on $A=M_{n}(E)$, one for each type.

For the case of characteristic 2 we will also use the notion of quadratic pair which extends the distinction between quadratic forms and symmetric bilinear forms to non-split central simple algebras. A quadratic pair on a central simple $F$-algebra $A$ is given by a couple $(\sigma, f)$ where $\sigma$ is a symplectic involution (we assume char $F=2$ ) and $f: \operatorname{Sym}(A, \sigma) \rightarrow F$ is an $F$-linear map subject to the condition $f(x+\sigma(x))=$ $\operatorname{Trd}_{A}(x)$ for all $x \in A$.

We want to compute the essential dimension of (conjugacy classes) of involutions and of quadratic pairs. These are the essential dimensions of the functors $\mathbf{U I n v}_{A}, \mathbf{O I n v}_{A}, \mathbf{S I n v}_{A}, \mathbf{Q P a i r s}_{A}:$ Fields $/ F \rightarrow$ Sets which takes a field extension $L / F$ to the set of conjugacy classes of unitary $L$-linear involutions, resp. orthogonal involutions, symplectic involutions and quadratic pairs on $A_{L}=A \otimes_{F} L$.

Example 3.1. Let $Q=(a, b)_{L}$ be a quaternion algebra and denote by $\gamma$ the canonical involution on $Q$. Let $L / F$ be a field extension. The only symplectic involution on $Q_{L}$ is $\gamma_{L}$. Hence ed $\operatorname{SInv}_{Q}=0$.

Every orthogonal involution $\sigma$ on $Q_{L}$ is of the form $\sigma=\operatorname{Int}(s) \circ \gamma_{L}$ for some $s \in \operatorname{Skew}\left(Q_{L}, \gamma_{L}\right) \backslash L$. The conjugacy class of $\sigma$ is determined uniquely by the discriminant of $\sigma$ [KMRT98, Example 7.4], given by $\operatorname{disc} \sigma=s^{2} \cdot\left(L^{\times}\right)^{2} \in L^{\times} /\left(L^{\times}\right)^{2}$. When $Q$ is split every element of $L^{\times}$is the square of some $s \in \operatorname{Skew}\left(Q_{L}, \gamma_{L}\right) \backslash L$, hence $\operatorname{OInv}_{Q}$ is isomorphic to the functor $H^{1}\left(-, \mu_{2}\right): L \mapsto L^{\times} /\left(L^{\times}\right)^{2}$. This implies that $\operatorname{ed}_{2} \operatorname{OInv}_{Q}=\operatorname{ed} \operatorname{OInv}_{Q}=1$ for a split quaternion algebra $Q$. We will see that if $Q$ is non-split then $\operatorname{ed}_{2} \mathbf{O I n v}_{Q}=\operatorname{ed} \mathbf{O} \operatorname{Inv}_{Q}=2$.

Theorem 3.1. Let $n$ be a power of 2 and $A$ be a division algebra of degree $n$ over its center. Then:

(a) Assume that the center $K$ of $A$ is a quadratic separable field extension of $F$ and $N_{K / F}(A)$ is split (i.e., there exist F-linear unitary involutions on $A$ ). Then

$$
\operatorname{ed} \mathbf{U} \mathbf{I n v}{ }_{A}=\operatorname{ed}_{2} \mathbf{U} \operatorname{Inv}_{A}=n^{2}-1 .
$$

(b) Assume that $Z(A)=F$ and $A \otimes_{F} A$ is split (i.e., there exist orthogonal involutions on $A)$. Then

$$
\operatorname{ed}^{\operatorname{OInv}} \operatorname{In}_{A}=\operatorname{ed}_{2} \operatorname{OInv}_{A}=\frac{n(n+1)}{2}-1
$$

(c) Assume that $Z(A)=F, A \otimes_{F} A$ is split and $n>1$ (i.e., there exist symplectic involutions on $A)$. Then

$$
\operatorname{ed} \mathbf{S I n v} \mathbf{v}_{A}=\operatorname{ed}_{2} \mathbf{S I n v}_{A}=\frac{n(n-1)}{2}-1
$$


(d) Assume that char $F=2, Z(A)=F, A \otimes_{F} A$ is split and $n>1$ (i.e., there exist quadratic pairs on $A)$. Then

$$
\text { ed QPairs }{ }_{A}=\operatorname{ed}_{2} \text { QPairs }_{A}=\frac{n(n+1)}{2}-1 .
$$

Proof. The functors $\mathbf{U I n v}_{A}, \mathbf{O I n v}_{A}$ and $\mathbf{S I n v}_{A}$ when $\sigma$ is a unitary $F$-linear, orthogonal, resp. symplectic involution are isomorphic to the functors

$$
H^{1}(-, \operatorname{Sim}(A, \sigma)): \text { Fields } / F \rightarrow \text { Sets, }
$$

where $\operatorname{Sim}(A, \sigma)$ is the group scheme of similitudes of $(A, \sigma)$, see [KMRT98, §29.D]. Similarly the functor QPairs $_{A}$ is isomorphic to the functor $H^{1}(-, \operatorname{Sim}(A, \sigma, f))$ when $(\sigma, f)$ is a quadratic pair on $A$. Notice that

$$
\operatorname{dim} \operatorname{Sim}(A, \sigma)= \begin{cases}n^{2}+1 & \text { if } \sigma \text { is unitary, } \\ \frac{n(n-1)}{2}+1 & \text { if } \sigma \text { is orthogonal, } \\ \frac{n(n+1)}{2}+1 & \text { if } \sigma \text { is symplectic. }\end{cases}
$$

and

$$
\operatorname{dim} \operatorname{Sim}(A, \sigma, f)=\frac{n(n-1)}{2}+1
$$

So in fact we need to show that

$$
\operatorname{ed} \operatorname{Sim}(A, \sigma)=\operatorname{ed}_{2} \operatorname{Sim}(A, \sigma)=\operatorname{dim}_{F} A-\operatorname{dim} \operatorname{Sim}(A, \sigma)
$$

for an involution $\sigma$ (of arbitrary type) on $A$, and similarly we must show that

$$
\text { ed } \operatorname{Sim}(A, \sigma, f)=\operatorname{ed}_{2} \operatorname{Sim}(A, \sigma, f)=\operatorname{dim}_{F} A-\operatorname{dim} \operatorname{Sim}(A, \sigma, f) .
$$

The group $\operatorname{Aut}_{K}(A, \sigma)$ naturally embeds in $\operatorname{Aut}_{K}(A)$ and $\operatorname{Sim}(A, \sigma)$ is the inverse image of $\mathbf{A u t}_{K}(A, \sigma)$ under Int: $R_{K / F}\left(\mathbf{G L}_{1}(A)\right) \rightarrow \mathbf{A u t}_{K}(A)$. The image of the map

$$
H^{1}\left(L, \mathbf{A u t}_{K}(A, \sigma)\right) \rightarrow H^{1}\left(L, \operatorname{Aut}_{K}(A)\right)
$$

consists of those isomorphism classes of Azumaya $K \otimes_{F} L$-algebras which admit an $L$-linear involution of the same type as $\sigma$. Similarly the image of the map $H^{1}\left(L, \mathbf{A u t}_{F}(A, \sigma, f)\right) \rightarrow H^{1}\left(L, \mathbf{A u t}_{F}(A)\right)$ consists of those isomorphism classes of central simple $L$-algebras that admit a quadratic pair. In view of Corollary 2.1 it suffices to show that the algebra $B=\left(a_{1}, b_{1}\right)_{L} \otimes_{L} \cdots \otimes_{L}\left(a_{r}, b_{r}\right)_{L} \otimes_{F} K$ from Corollary 2.1 admits an involution of the same type as $\sigma$, resp. a quadratic pair. This is easy to see directly, and can also be proven by looking at $B \otimes_{F} B$ (when $K=F)$, resp. $N_{K / F}(B)$ (when $[K: F]=2$ ). Hence the claim follows.

\section{Subalgebras}

Let $A$ be a central simple $F$-algebra and $E$ be a subalgebra of $A$. We call $(A, E)$ a couple of $F$-algebras. If $\left(A^{\prime}, E^{\prime}\right)$ is another couple of $F$-algebras we say that $(A, E)$ and $\left(A^{\prime}, E^{\prime}\right)$ are isomorphic if there exists an isomorphism $\varphi: A \stackrel{\sim}{\rightarrow} A^{\prime}$ of $F$-algebras which restricts to an isomorphism $E \stackrel{\sim}{\rightarrow} E^{\prime}$. We call two subalgebras $E$ and $E^{\prime}$ of $A$ conjugate if $(A, E)$ and $\left(A, E^{\prime}\right)$ are isomorphic. Equivalently there exists $a \in A^{\times}$ such that $E^{\prime}=a E a^{-1}$

Let $G$ be the inverse image of $H=\mathbf{A u t}_{F}(A, E)$ under Int: $\mathbf{G L}_{1}(A) \rightarrow \mathbf{A u t}_{F}(A)$. It is the normalizer of $\mathbf{G L}_{1}(E)$ in $\mathbf{G L}_{1}(A)$. We will compute the essential dimension of $G$ in some examples. For this section we use [KMRT98, §29.C] as a reference for 
cohomological statements. We start with an additional result, which gives us an interpretation of the functor $H^{1}(-, G)$ :

Lemma 4.1. The set $H^{1}(L, G)$ is naturally (in $L / F$ ) isomorphic to the set of conjugacy classes of $L$-subalgebras $E^{\prime}$ of $A_{L}$ such that $E \otimes_{F} L_{\mathrm{alg}}$ and $E^{\prime} \otimes_{L} L_{\mathrm{alg}}$ are conjugate subalgebras of $A \otimes_{F} L_{\mathrm{alg}}=A_{L} \otimes_{L} L_{\mathrm{alg}}$.

Proof. Let $L / F$ be a field extension. The set $H^{1}\left(L, \mathbf{A u t}_{F}(A, E)\right)$ is in one-toone correspondence with the set of $F$-isomorphism classes of couples of $L$-algebras $\left(A^{\prime}, E^{\prime}\right)$ such that $\left(A^{\prime}, E^{\prime}\right)_{\text {alg }} \simeq\left(A_{L}, E_{L}\right)_{\mathrm{alg}}$.

We have an exact sequence $1 \rightarrow \mathbf{G}_{m} \rightarrow G \rightarrow \mathbf{A u t}_{F}(A, E) \rightarrow 1$. The map $H^{1}(L, G) \rightarrow H^{1}\left(L, \operatorname{Aut}_{F}(A, E)\right)$ is injective, since $H^{1}\left(L, \mathbf{G}_{m}\right)$ is trivial and $\mathbf{G}_{m}$ lies in the center of $G$. Hence $H^{1}(L, G)$ is naturally (in $L / F$ ) bijective to the kernel of the connecting map $H^{1}\left(L, \mathbf{A u t}_{F}(A, E)\right) \rightarrow H^{2}\left(L, \mathbf{G}_{m}\right)=\operatorname{Br}(L)$, which takes a couple $\left(A^{\prime}, E^{\prime}\right)$ to the Brauer class of $A^{\prime} \otimes_{F} A^{\text {op }}$. Thus $H^{1}(L, G)$ ist the set of isomorphism classes of couples of $L$-algebras $\left(A^{\prime}, E^{\prime}\right)$ such that $A^{\prime} \simeq A_{L}$ and $\left(A^{\prime}, E^{\prime}\right)_{\mathrm{alg}} \simeq\left(A_{L}, E_{L}\right)_{\mathrm{alg}}$. The map which associates to the conjugacy class of an $L$ subalgebra $E^{\prime}$ on $A_{L}$ the isomorphism class of the couple $\left(A_{L}, E^{\prime}\right)$ then establishes a (well defined and in $L / F$ natural) bijection between the set of conjugacy classes of $L$-subalgebras $E^{\prime}$ of $A_{L}$ such that $E^{\prime} \otimes_{L} L_{\text {alg }}$ is conjugate to $E \otimes_{F} L_{\text {alg }}$ and the set $H^{1}(L, G)$.

4.1. Étale subalgebras. In this subsection we try to compute the essential dimension of the functor Fields $/ F \rightarrow$ Sets taking a field $L$ to the set of conjugacy classes of étale subalgebras $E$ of a given type of $A_{L}$, where $A$ is a fixed central simple $F$-algebra.

The type of an étale subalgebra $E$ of a central simple algebra $B$ is defined as follows. Denote by $L$ the center of $B$ and let $e_{1}, \ldots, e_{d}$ be the minimal non-zero idempotents of $E \otimes_{F} L_{\text {sep }}$, where $d=\operatorname{dim} E$. Write $B_{\text {sep }}=B \otimes_{L} L_{\text {sep }}$. Let $r_{i}=$ $\operatorname{rdim} e_{i} B_{\text {sep }}=\frac{\operatorname{dim} e_{i} B_{\text {sep }}}{\operatorname{deg} B} \in \mathbb{N}$ be the reduced dimension of the right ideal $e_{i} B_{\text {sep }}$. Then the multiset $t_{E}=\left[r_{1}, \ldots, r_{d}\right]$ is uniquely determined by $E$ and is invariant under conjugation (of étale subalgebras) and scalar extension. The invariant $t_{E}$ is a partition of $\operatorname{deg} B$, i.e., $\sum_{i=1}^{d} r_{d}=\operatorname{deg} B$. It was introduced by D. Krashen [Kr04], who called it the type of the étale subalgebra $E$ of $B$.

In case $A=M_{n}(F)$ there exists for every partition $t=\left[r_{1}, \ldots, r_{d}\right]$ of $\operatorname{deg} A=n$ an étale subalgebra $E$ of $A$ of type $t_{E}=t$. Moreover over a separably closed field $F$ every étale subalgebra of $A=M_{n}(F)$ of given type $t=\left[r_{1}, \ldots, r_{d}\right]$ is conjugate to the étale subalgebra $E=\bigoplus_{i=1}^{d} F d_{i}$ where $d_{i}$ is the diagonal matrix with ones from place $s_{i}+1:=\sum_{j=1}^{i-1} r_{j}+1$ up to place $s_{i+1}$ and zeros on the other places. This follows from the fact that commuting idempotent matrices are simultaneously diagonalizable.

Consider the functor $\mathbf{E}_{A}^{(t)}:$ Fields $/ F \rightarrow$ Sets, which takes a field extension $L / F$ to the set of conjugacy classes of étale subalgebras of $A_{L}$ of type $t$.

Example 4.1. Let $A$ be a central simple algebra of degree $n$ and let $t=[1, \ldots, 1]$ (with $n$ ones). Then the functors $\mathbf{E}_{A}^{(t)}$ is isomorphic to the functor taking a field $L \in$ Fields $/ F$ to the set of isomorphism classes of $n$-dimensional étale $L$-algebras that embed in $A_{L}$. In fact two $n$-dimensional étale subalgebras of $A_{L}$ are conjugate if and only if they are isomorphic. 
We will consider the case when $A$ is a division algebra. Every étale subalgebra $E$ of a division algebra is a field and $d=\operatorname{dim}_{F} E$ divides $n=\operatorname{deg} A$. Moreover every (étale) subfield of dimension $d$ has type $\left[\frac{n}{d}, \ldots, \frac{n}{d}\right]$, since the absolute Galois group of $F$ permutes the idempotents in $E_{\mathrm{sep}}=\bigoplus_{i=1}^{d} F_{\text {sep }} e_{i}$ transitively.

Theorem 4.1. Let $A$ be a division F-algebra of degree $n=\operatorname{deg} A=p^{r}$ a power of $p$. Let $d=p^{s}$ divide $n$ and set $t=\left[\frac{n}{d}, \ldots, \frac{n}{d}\right]$ (the multiset with $d$ identical elements). Assume that $A$ admits an étale subalgebra of type $t$. Then

$$
\text { ed } \boldsymbol{E}^{(t)}=\operatorname{ed}_{p} \dot{\boldsymbol{E}} \boldsymbol{t}_{A}^{(t)}=n^{2}-\frac{n^{2}}{d}=p^{2 r}-p^{2 r-s}
$$

Proof. Let $E$ be an étale subalgebra of $A$ of type $t$. Let $H=\mathbf{A u t}_{F}(A, E)$ and $G=$ $\operatorname{Int}^{-1}(H)$ its inverse image under Int: $\mathbf{G L}_{1}(A) \rightarrow \mathbf{A u t}_{F}(A)$. It is the normalizer of the torus $R_{E / F}\left(\mathbf{G}_{m}\right)$ in $\mathbf{G L}_{1}(A)$. By Lemma 4.1 the functors $H^{1}(-, G)$ and $\mathbf{E}^{(t)}$ are isomorphic.

We can pass to an algebraic closure in order to compute the dimension of $G$. Then $G$ is the normalizer of $R_{E / F}\left(\mathbf{G}_{m}\right)$ in $\mathbf{G L}_{n}$ and $G^{0}$ is the centralizer of $R_{E / F}\left(\mathbf{G}_{m}\right)$ in $\mathbf{G L}_{n}$. We may replace $E$ by the conjugate subalgebra $E^{\prime}=\sum_{i} F d_{i}$, where $d_{i}$ is the diagonal matrix with ones from position $(i-1) p^{r-s}+1$ to $i p^{r-s}$ and zeros otherwise. Then $G^{0}=\mathbf{G L}_{p^{r-s}} \times \cdots \times \mathbf{G L}_{p^{r-s}} \subseteq \mathbf{G L}_{n}$ has dimension $p^{s} \cdot p^{2(r-s)}=p^{2 r-s}$. It follows that $\operatorname{dim} G=p^{2 r-s}$.

The image of $H^{1}\left(L, \mathbf{A u t}_{F}(A, E)\right) \rightarrow H^{1}\left(L, \mathbf{A u t}_{F}(A)\right)$ consists of those isomorphism classes of central simple $L$-algebras that admit an étale subalgebra of type $t$. In view of Corollary 2.1 it suffices to show that the division $F$-algebra $B=\left(a_{1}, b_{1}\right)_{L} \otimes_{L} \cdots \otimes_{L}\left(a_{r}, b_{r}\right)_{L}$ from Corollary 2.1 admits an étale subalgebra of type $t$. This is true, since we can take for $E$ the tensor product $E_{1} \otimes_{L} \cdots \otimes_{L} E_{s}$ where $E_{1}, \ldots, E_{s}$ are maximal subfields of $\left(a_{1}, b_{1}\right)_{L}, \ldots,\left(a_{s}, b_{s}\right)_{L}$ respectively.

Remark 4.1. In the extreme cases $t=[1, \ldots, 1]$ and $t=[n]$ we can also compute the essential $p$-dimension of $\mathbf{E} \mathbf{t}_{A}^{(t)}$ for a split algebra $A=M_{n}(F)$ of degree $n$ assuming char $F \neq p$ : We have $\operatorname{ed}_{p} \mathbf{E} \mathbf{t}_{M_{n}(F)}^{[n]}=0$ and

$$
\operatorname{ed}_{p} \dot{\mathbf{E}} \mathbf{t}_{M_{n}(F)}^{[1, \ldots, 1]}=\operatorname{ed}_{p} S_{n}=\left\lfloor\frac{n}{p}\right\rfloor
$$

The first statement is trivial since the only 1-dimensional étale subalgebra of $A$ is $F$. The functors $\mathbf{E} \mathbf{t}_{M_{n}(F)}^{[1, \ldots, 1]}$ and $H^{1}\left(-, S_{n}\right)$ are isomorphic by Example 4.1, since every $n$-dimensional étale algebra embeds in $M_{n}(F)$. This $\operatorname{shows}_{p} \operatorname{ed}_{p} \mathbf{E}_{M_{n}(F)}^{[1, \ldots, 1]}=\operatorname{ed}_{p} S_{n}$. The equality $\operatorname{ed}_{p} S_{n}=\left\lfloor\frac{n}{p}\right\rfloor$ (when $\operatorname{char} F \neq p$ ) was first noticed by J.-P. Serre, see [MR08, Corollary 4.2].

In contrast the (absolute) essential dimension of $\mathbf{E t}_{M_{n}(F)}^{[1, \ldots, 1]}$, being equal to the essential dimension of $S_{n}$, is unknown for $n \geq 8$.

4.2. Central simple subalgebras. Let $A$ be a central simple $F$-algebra and let $d$ be a divisor of $\operatorname{deg} A$. Consider the functor $\mathbf{S u b}_{A}^{(d)}:$ Fields $/ F \rightarrow$ Sets, which takes a field extension $L / F$ to the set of isomorphism classes of central simple $L$-subalgebras of $A_{L}$ of degree $d$. 
Example 4.2. When $\operatorname{deg} A$ is divisible by $d^{2}$ and ind $A$ divides $\frac{\operatorname{deg} A}{d^{2}}$ the functor $\mathbf{S u b}_{A}^{(d)}$ is isomorphic to the functor $\mathbf{C S A}_{d}$, taking a field extension $L / F$ to the set of isomorphism classes of central simple $L$-algebras of degree $d$. In fact every central simple algebra $B$ over $L$ of degree $d$ is a subalgebra of $B \otimes_{L} B^{\text {op }} \simeq M_{d^{2}}(L)$, hence of $A_{L}$.

Theorem 4.2. Let $n=p^{r}$ for some $r \geq 0$ and let $A$ be a division algebra of degree $n$. Let $d=p^{s}$ be a divisor of $n$ and assume that $A$ admits a central simple subalgebra of degree $d$. Then

$$
\operatorname{ed}_{\mathbf{S u b}}^{(d)}=\operatorname{ed}_{p} \mathbf{S u b}_{A}^{(d)}=n^{2}-d^{2}-\frac{n^{2}}{d^{2}}+1=p^{2 r}-p^{2 s}-p^{2(r-s)}+1
$$

Proof. Let $B$ be a central simple subalgebra of $A$ of degree $d$. Set $H=\mathbf{A u t}_{F}(A, B)$ and $G=\operatorname{Int}^{-1}(H)$ the inverse image of $H$ under $\operatorname{Int:} \mathbf{G L}_{1}(A) \rightarrow \mathbf{A u t}_{F}(A)$. It is the normalizer of $\mathbf{G} \mathbf{L}_{1}(B)$ in $\mathbf{G L}_{1}(A)$.

I claim that $\mathbf{S u b}_{A}^{(d)}$ is isomorphic to $H^{1}(-, G)$. In fact by Lemma 4.1 the set $H^{1}(L, G)$ is in natural one-to-one correspondence with the set of conjugacy classes of central simple subalgebras $B^{\prime}$ of $A_{L}$ such that $B^{\prime} \otimes_{L} L_{\text {sep }}$ and $B \otimes_{F} L_{\text {sep }}$ are conjugate subalgebras of $A \otimes_{F} L_{\text {sep }}$. By the Skolem Noether theorem two central simple subalgebras of a central simple algebra are conjugate if and only if they are isomorphic. Hence the claim follows.

Since the division $F$-algebra $B=\left(a_{1}, b_{1}\right)_{L} \otimes_{L} \cdots \otimes_{L}\left(a_{r}, b_{r}\right)_{L}$ from Corollary 2.1 admits a central simple subalgebra of degree $d$ we obtain

$$
\operatorname{ed} G=\operatorname{ed}_{p} G=n^{2}-\operatorname{dim} G
$$

as in the proof of Theorem 4.1.

Let $C$ denote the centralizer of $B$ in $A$. Then $G$ fits into an exact sequence

$$
1 \rightarrow \mathbf{G L}_{1}(C) \rightarrow G \rightarrow \operatorname{Aut}_{F}(B) \rightarrow 1 \text {. }
$$

It follows, that $G$ has dimension

$$
\operatorname{dim} G=\frac{n^{2}}{d^{2}}+d^{2}-1
$$

This concludes the proof.

\section{ESSENTIAL DIMENSION OF RELATED GROUP SCHEMES}

In section 3 we have computed the essential dimension of the group scheme $\operatorname{Sim}(A, \sigma)$ for certain algebras with involution $(A, \sigma)$. The essential dimension of the group scheme $\mathbf{I s o}(A, \sigma)$ of isometries of $(A, \sigma)$ is closely related:

Proposition 5.1. Let $(A, \sigma)$ be a central simple F-algebra with involution. Then ed $\operatorname{Iso}(A, \sigma) \leq \operatorname{ed} \operatorname{Sim}(A, \sigma)+1$. Moreover if $\sigma$ is unitary or char $F \neq 2$ then ed $\operatorname{Iso}(A, \sigma)$ is either equal to ed $\operatorname{Sim}(A, \sigma)$ or $\operatorname{ed} \operatorname{Sim}(A, \sigma)+1$.

Furthermore the analogous statements hold for essential $p$-dimension for every prime $p$.

Proof. The first inequality follows from [Me09, Corollary 4.3] and the fact that Iso $(A, \sigma)$ has codimension 1 in $\operatorname{Sim}(A, \sigma)$.

If $\sigma$ is unitary or if char $F \neq 2$ there is a surjection of functors

$$
H^{1}(-, \mathbf{I s o}(A, \sigma)) \rightarrow H^{1}(-, \operatorname{Sim}(A, \sigma))
$$


(cf. [KMRT98, §29.D and Propositions 2.7 and 2.18]). Hence ed $\operatorname{Sim}(A, \sigma) \leq \operatorname{ed} \operatorname{Iso}(A, \sigma)$ by [BF03, Lemma 1.9] and similarly $\operatorname{ed}_{p} \operatorname{Sim}(A, \sigma) \leq \operatorname{ed}_{p} \operatorname{Iso}(A, \sigma)$.

For division algebras of degree a power of 2 we can say more:

Proposition 5.2. Let $n=2^{r}$ for some $r \geq 0$ and let $(A, \sigma)$ be a central simple $F$-algebra with involution, where $A$ is a division algebra of degree $n$. Then

- If $\sigma$ is unitary then $n^{2}-1 \leq \operatorname{ed}_{2} \mathbf{U}(A, \sigma) \leq \operatorname{ed} \mathbf{U}(A, \sigma) \leq n^{2}$.

- If $\sigma$ is orthogonal then $\operatorname{ed} \mathbf{O}(A, \sigma)=\operatorname{ed}_{2} \mathbf{O}(A, \sigma)=\frac{n(n+1)}{2}$.

- If $\sigma$ is symplectic then $\operatorname{ed} \mathbf{S p}(A, \sigma)=\operatorname{ed}_{2} \mathbf{S p}(A, \sigma)=\frac{n^{2}(n-1)}{2}$.

- If $(\sigma, f)$ is a quadratic pair then $\operatorname{ed} \mathbf{O}(A, \sigma, f)=\operatorname{ed}_{2} \mathbf{O}(A, \sigma, f)=\frac{n(n+1)}{2}$. Moreover in case $r \geq 2$ (and char $F=2$ ): $\operatorname{ed}^{+}(A, \sigma, f)=\operatorname{ed}_{2} \mathbf{O}^{+}(A, \sigma, f)=$ $\frac{n(n+1)}{2}$ and $\operatorname{ed}_{\mathbf{G O}}{ }^{+}(A, \sigma, f)=\operatorname{ed}_{2} \mathbf{G O}^{+}(A, \sigma, f)=\frac{n(n+1)}{2}-1$.

Remark 5.1. The assumption $r \geq 2$ in the statements about $\mathbf{G O}^{+}(A, \sigma, f)$ and $\mathbf{O}^{+}(A, \sigma, f)$ is needed. When $r=1$ the algebra $A$ is a quaternion division algebra, $A=(a, b)_{F}, \sigma$ its canonical involution, and I claim that ed $\mathbf{G O}^{+}(A, \sigma, f)=$ $0<2$ and $\operatorname{ed} \mathbf{O}^{+}(A, \sigma, f)=1<3$. In fact $\mathbf{G O}^{+}(A, \sigma, f)=R_{F(u) / F}\left(\mathbf{G}_{m}\right)$ and $\mathbf{O}^{+}(A, \sigma, f)=R_{F(u) / F}^{(1)}\left(\mathbf{G}_{m}\right)$ are tori. Their essential dimension and 2-dimension were computed in [LMMR10] (cf. [BF03, Theorem 2.5]).

Proof. The statements about $\mathbf{U}(A, \sigma)$ follow from Theorem 3.1 and Proposition 5.1.

For orthogonal and symplectic involutions the inequalities ed $\operatorname{ed}_{2} \mathbf{O}(A, \sigma) \leq \operatorname{ed} \mathbf{O}(A, \sigma) \leq$ $\frac{n(n+1)}{2}$ and $\operatorname{ed}_{2} \mathbf{S p}(A, \sigma) \leq \operatorname{ed} \mathbf{S p}(A, \sigma) \leq \frac{n(n-1)}{2}$ are clear. In order to show the reverse inequalities we slightly refine the approach given in section 2 . We have a commutative diagram

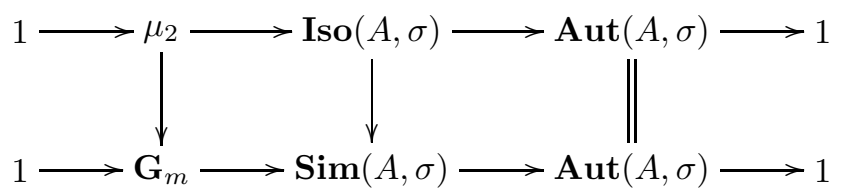

with exact rows, where the columns are closed embeddings. By [Me09, Example 3.6 and Theorem 4.8] (cf. [BRV08, Corollary 4.2])

$$
\operatorname{ed}_{2} \operatorname{Iso}(A, \sigma) \geq \operatorname{ind}\left(\mathbf{I s o}(A, \sigma), \mu_{2}\right)-\operatorname{dim} \operatorname{Iso}(A, \sigma),
$$

where $\operatorname{ind}\left(\mathbf{I s o}(A, \sigma), \mu_{2}\right)$ is the maximal index of the image in $H^{2}\left(L, \mu_{2}\right)=\operatorname{Br}_{2}(L)$ of an $H$-torsor, taken over all $H$-torsors over $L$ and all field extensions $L / F$. From the above diagram it is clear that $\operatorname{ind}\left(\operatorname{Iso}\left(A, \sigma, \mu_{2}\right)=\operatorname{ind}\left(\operatorname{Sim}(A, \sigma), \mathbf{G}_{m}\right)\right.$. As shown in the proof of Theorem 3.1 the latter equals $\operatorname{dim}_{F} A$. Since $\operatorname{dim}_{F} A-\operatorname{dim} \mathbf{O}(A, \sigma)=$ $\frac{n(n+1)}{2}$ and $\operatorname{dim}_{F} A-\operatorname{dim} \mathbf{S p}(A, \sigma)=\frac{n(n-1)}{2}$ the claim follows.

With a similar argument one gets ed $\mathbf{O}(A, \sigma, f)=\operatorname{ed}_{2} \mathbf{O}(A, \sigma, f)=\frac{n(n+1)}{2}$ for a quadratic pair $(\sigma, f)$. The groups $\mathbf{O}^{+}(A, \sigma, f)$ and $\mathbf{G O}^{+}(A, \sigma, f)$ have finite index in $\mathbf{O}(A, \sigma, f)$ and $\mathbf{G O}(A, \sigma, f)$, respectively. Hence ed $\mathbf{O}^{+}(A, \sigma, f) \leq \frac{n(n+1)}{2}$ and ed $\mathbf{G O}^{+}(A, \sigma, f) \leq \frac{n(n+1)}{2}-1$. In order to show the reverse inequalities for $r \geq 2$ 
we look at the diagram

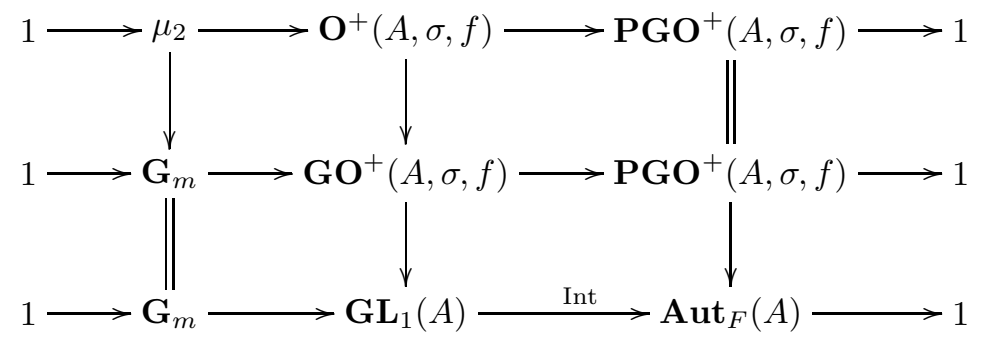

with exact rows. The image of the map $H^{1}\left(L, \mathbf{P G O}^{+}(A, \sigma, f)\right) \rightarrow H^{1}\left(L, \mathbf{A u t}_{F}(A)\right)$ consists of the classes of central simple $L$-algebras $A^{\prime}$ of degree $\operatorname{deg} A^{\prime}=\operatorname{deg} A$ which admit a quadratic pair $\left(\sigma^{\prime}, f^{\prime}\right)$ such that $\operatorname{disc}\left(\sigma_{L}, f_{L}\right)=\operatorname{disc}\left(\sigma^{\prime}, f^{\prime}\right)$. By [Be05, Theorem 2] (note that char $L=2$ ) every central simple $L$-algebra $A^{\prime}$ which is not a quaternion division algebra, and which has a symplectic involution, admits quadratic pairs of arbitrary discriminant. In particular the algebra $B=$ $\left(a_{1}, b_{1}\right) \otimes_{L} \cdots \otimes_{L}\left(a_{n}, b_{n}\right)$ from Corollary 2.1 (with $p=2$ ) admits quadratic pairs of arbitrary discriminant. Therefore the inequality $\operatorname{ed}_{2} \mathbf{G O}^{+}(A, \sigma, f) \geq \frac{n(n+1)}{2}-1$ is a consequence of Corollary 2.1 and the inequality $\operatorname{ed}_{2} \mathbf{O}^{+}(A, \sigma, f) \geq \frac{n(n+1)}{2}$ follows from [Me09, Example 3.6 and Theorem 4.8].

Remark 5.2. Suppose the following statement holds true: The essential dimension ed $\chi$ of every gerbe $\chi$ banded by $R_{K / F}^{(1)}\left(\mathbf{G}_{m}\right)$ is equal to cdim $\chi+1$ (for the notion of gerbes and their essential dimension see [BRV08]). Then one can show that ed $U(A, \sigma)=n^{2}$ for a central division $K$-algebra $A$ of degree $n$ a power of 2 and a unitary $F$-linear involution $\sigma$ on $A$.

\section{ACKNowledgments}

I am grateful to Z. Reichstein for helpful comments.

\section{REFERENCES}

[Be05] G. Berhuy, Erratum to: "On the set of discriminants of quadratic pairs" [JPAA 188 (2004) 33-44], J. Pure Appl. Algebra 195 (2005), 125-126.

[BF03] G. Berhuy, G. Favi, Essential dimension: A functorial point of view (after A. Merkurjev), Doc. Math. 8 (2003), 279-330 (electronic).

[BR05] G. Berhuy, Z. Reichstein, On the notion of canonical dimension of algebraic groups, Adv. Math. 198 (2005), 128-171.

[BRV08] P. Brosnan, Z. Reichstein, A. Vistoli, Essential dimension of moduli of curves and other algebraic stacks, arxiv:0907.0924.v2 [math.AG] (October 6, 2009).

[Ka00] N.A. Karpenko, On anisotropy of orthogonal involutions. J. Ramanujan Math. Soc. 15(1) (2000), 1-22.

[Ka09] N.A. Karpenko, Incompressibility of quadratic Weil transfer of generalized Severi-Brauer varieties. Linear Algebraic Groups and Related Structures (preprint server) 362 (2009, Oct 28), 12 pages.

[KMRT98] M.-A. Knus, A. Merkurjev, M. Rost, and J.-P. Tignol, The book of involutions, American Math. Soc. Providence, RI, (1998), With a preface in French by J. Tits.

[Kr04] D. Krashen, Zero cycles on homogeneous varieties, Adv. Math. 223, (2010), no. 6, 20222048.

[LMMR10] R. Lötscher, M. MacDonald, A. Meyer, and Z. Reichstein, Essential dimension of algebraic tori. Linear Algebraic Groups and Related Structures (preprint server) 399 (2010, Aug 17), 12 pages. 
[Me09] A.S. Merkurjev, Essential dimension, in Quadratic forms - algebra, arithmetic, and geometry (R. Baeza, W.K. Chan, D.W. Hoffmann, and R. Schulze-Pillot, eds.), Contemporary Mathematics 493 (2009), 299-326.

[JW90] B. Jacob and A. Wadsworth, Division algebras over Henselian fields, J. Algebra 128 (1990), no. 1, 126-179.

[Me10] A.S. Merkurjev Essential dimension of PGL $\left(p^{2}\right)$, J. Amer. Math. Soc. 23, (2010), 693-712.

[MR08] A. Meyer, Z. Reichstein The essential dimension of the normalizer of a maximal torus in the projective linear group, Algebra and Number Theory 3 (2009), No. 4, 467-487.

[Ti78] J.-P. Tignol, Sur les classes de similitude de corps à involution de degrée 8, C. R. Acad. Sci. Paris Sér. A-B 286 (1978), no. 20, A875-A876.

[Wa79] W.C. Waterhouse, Introduction to affine group schemes, Springer, New York (1979).

ROLAND LÖTSCHER

Mathematisches Institut Der Ludwig-Maximilians-Universität MünChen,

Theresienstrasse 39, D-80333 MünChen, Germany

E-mail address: Roland.Loetscher@mathematik.uni-muenchen.de 